\title{
Modulation of Neural Oscillatory Activity during Dynamic Face Processing
}

\author{
Elaine Foley, Gina Rippon, and Carl Senior
}

\begin{abstract}
Various neuroimaging and neurophysiological methods have been used to examine neural activation patterns in response to faces. However, much of previous research has relied on static images of faces, which do not allow a complete description of the temporal structure of face-specific neural activities to be made. More recently, insights are emerging from fMRI studies about the neural substrates that underpin our perception of naturalistic dynamic face stimuli, but the temporal and spectral oscillatory activity associated with processing dynamic faces has yet to be fully characterized. Here, we used MEG and beamformer source localization to examine the spatiotemporal profile of neurophysiological oscillatory activity in response to dynamic faces.
\end{abstract}

\section{INTRODUCTION}

The ability to recognize faces and process expressed intentions and emotions is at the center of human social perception skills. Various neuroimaging and neurophysiological methods have been used to examine neural activation patterns in response to a diverse range of face processing tasks, commonly using static images of faces. These methods have informed the development of valuable detailed cortical network models of face perception (Ishai, 2008; Haxby, Hoffman, \& Gobbini, 2002), and the significant contribution of these techniques to our understanding of face perception has been widely documented elsewhere (Vuilleumier \& Pourtois, 2007; Calder \& Young, 2005; Haxby, Hoffman, \& Gobbini, 2000). However, it is important to consider that faces are inherently dynamic rather than static and are most often seen moving in the real world. Yet, previous studies investigating face processing have overwhelmingly relied on static images of faces. These posed static stimuli do not allow a complete description of the temporal structure of facespecific neural activities to be made (Adolphs, 2002b), as they represent impoverished displays lacking natural facial motion (Ambadar, Schooler, \& Cohn, 2005). Dynamic face stimuli therefore offer a more suitable means of examining the neural basis of realistic natural face perception.

Aston University, Birmingham, UK
Source analysis revealed a number of regions showing enhanced activation in response to dynamic relative to static faces in the distributed face network, which were spatially coincident with regions that were previously identified with fMRI. Furthermore, our results demonstrate that perception of realistic dynamic facial stimuli activates a distributed neural network at varying time points facilitated by modulations in low-frequency power within alpha and beta frequency ranges $(8-30 \mathrm{~Hz})$. Naturalistic dynamic face stimuli may provide a better means of representing the complex nature of perceiving facial expressions in the real world, and neural oscillatory activity can provide additional insights into the associated neural processes.

In recent years, insights are emerging from neuroimaging studies that have used fMRI to explore the neural substrates involved in processing naturalistic dynamic face stimuli. These studies have revealed differential patterns of activation for dynamic and static faces (Foley, Rippon, Thai, Longe, \& Senior, 2012; Pitcher, Dilks, Saxe, Triantafyllou, \& Kanwisher, 2011; Fox, Iaria, \& Barton, 2009; LaBar, Crupain, Voyvodic, \& McCarthy, 2003). In general, explicit movement information in dynamic face stimuli has been shown to activate a richer and partly different, broader network of regions compared with static stimuli (Foley et al., 2012; Fox et al., 2009; Schultz \& Pilz, 2009; Trautmann, Fehr, \& Herrmann, 2009). Dynamic face stimuli have consistently been shown to elicit larger responses relative to static faces in the STSs (Foley et al., 2012; Pitcher et al., 2011; Fox et al., 2009; Schultz \& Pilz, 2009; Sato, Kochiyama, Yoshikawa, Naito, \& Matsumura, 2004; Kilts, Egan, Gideon, Ely, \& Hoffman, 2003; LaBar et al., 2003), middle temporal gyri (MTGs), and inferior frontal gyri, among others (Foley et al., 2012; Sato et al., 2004). These studies have harnessed the powerful spatial resolution of fMRI to localize the neural networks involved in processing dynamic faces but have yet to fully characterize the temporal and spectral information within these networks.

A handful of neurophysiological studies using EEG and MEG have explored spatiotemporal processing of dynamic face stimuli. Motion-sensitive ERPs have been identified with EEG over bilateral occipito-temporal 
electrodes when participants viewed dynamic images of the face, hand, and body (Wheaton, Pipingas, Silberstein, \& Puce, 2001). In a series of EEG studies, Puce, Smith, and Allison (2000) found a prominent negativity between 170 and $220 \mathrm{msec}$ (N170) in response to the apparent motion of a natural face over posterior scalp electrodes and to the movement of a line-drawn face (Puce \& Perrett, 2003). More recently, Johnston, Molyneux, and Young (2015) reported an increased N170 response to free viewing of ambient video stimuli of faces, providing support for the N170 response in a more ecologically valid setting. A corresponding sensor-level MEG response, the M170, has also been described to the apparent motion of natural faces (Watanabe, Kakigi, \& Puce, 2001) and more recently to facial avatars (Ulloa, Puce, Hugueville, \& George, 2014). Similarly, Watanabe, Miki, and Kakigi (2005) and Miki et al. (2004, 2007, 2011) found that the observation of different types of facial motion, including mouth and eye movements, elicited evoked responses with a peak latency of $160 \mathrm{msec}$ in lateral occipito-temporal cortex, corresponding to area MT (for a review, see Miki \& Kakigi, 2014).

At the source level, Sato, Kochiyama, Uono, and Yoshikawa (2008) described an increased evoked response to averted versus straight gazes, between 150 and $200 \mathrm{msec}$, which peaked at $170 \mathrm{msec}$, in the STS. More recently, Sato, Kochiyama, and Uono (2015) found that posterior regions including visual area V5, the fusiform gyrus, and the STS showed increased evoked activity in response to dynamic facial expressions relative to dynamic mosaic control stimuli between 150 and 200 msec after stimulus onset. Taken together, these findings present a relatively consistent picture, whereby facial motion elicits increased evoked activity around $200 \mathrm{msec}$ in occipito-temporal regions. These regions form part of the so-called "core system" in Haxby et al.'s (2000) model of face perception. According to this model, the perception of identity, the invariant aspect of a face, is believed to occur in the lateral fusiform gyrus at approximately $170 \mathrm{msec}$, whereas the STS is more involved in processing the changeable facial features, such as eye and mouth movements, around the same time (Pitcher et al., 2011; Haxby et al., 2000). Regions of the so-called "extended system" such as the amygdala, OFC, somatosensory cortex, and IC may be recruited from around $300 \mathrm{msec}$ after stimulus onset, to link the perceptual representation to conceptual knowledge of the emotional and social meanings of perceived expressions (Adolphs, 2002a).

Remarkably, relative to the wealth of neurophysiological studies that have examined the time course of evoked responses to faces, very few have explored the ongoing neural oscillatory activity associated with face processing, particularly with respect to dynamic facial displays. Evoked responses focus specifically on phase-locked neural responses to stimuli; however, this neural activity constitutes only part of the total neural response to a delivered stimulus (Donner \& Siegel, 2011; Hillebrand, Singh, Holliday, Furlong, \& Barnes, 2005). It is increasingly becoming clear that neural oscillations play an important role in brain function and the synchronized activity of oscillating neural networks is now believed to be the critical link between single-neuron activity and behavior (Buzsáki \& Draguhn, 2004; Engel, Fries, \& Singer, 2001). There is also a growing body of literature to suggest that neural oscillations are involved in supporting information transfer and binding between brain regions (Donner \& Siegel, 2011; Engel \& Fries, 2010; Buzsáki \& Draguhn, 2004). More recently, face processing has been linked to neural oscillatory activity in studies using various paradigms involving predominantly static face stimuli (Furl, Coppola, Averbeck, \& Weinberger, 2014; Schyns, Thut, \& Gross, 2011). We therefore examined ongoing neural oscillatory activity, or so-called induced oscillations, during dynamic face processing in this study to assess additional aspects of face perception that may not be evident in the modulation of the evoked response.

Unlike fMRI, the MEG signal provides a direct measure of electrical neural activity and is therefore well suited to the investigation of induced neural oscillatory responses, particularly when combined with beamforming source localization techniques such as synthetic aperture magnetometry (SAM). Beamformer methods can be used to examine temporal and spectral information at both short and longer latencies, which is particularly useful when exploring cognitive processes such as face perception (Hillebrand et al., 2005). Notably, the output of the beamformer can be described as a "virtual electrode," which may be visualized as time-frequency plots of activity arising from specific voxels where spectral power changes are identified (Hillebrand et al., 2005; Singh, Barnes, Hillebrand, Forde, \& Williams, 2002). This permits the detailed examination of temporal and spectral power changes within specific ROIs, in this case, within regions of the face perception network.

MEG and SAM source localization methods have been used to investigate changes in induced cortical oscillatory activity in response to viewing various dynamic biological motion stimuli (Muthukumaraswamy, Johnson, Gaetz, \& Cheyne, 2006; Singh et al., 2002). Singh et al. (2002) reported decreases in induced oscillatory power in 5- to 15and $15-$ to $25-\mathrm{Hz}$ frequency bands in response to viewing point light displays of biological motion in area MT and the STS. Similarly, Muthukumaraswamy et al. (2006) found decreases in low-frequency power, in alpha (8$15 \mathrm{~Hz}$ ) and beta $(15-35 \mathrm{~Hz})$ bands, and in sources within lateral sensorimotor areas and bilateral occipito-parietal regions in response to viewing dynamic biological motion stimuli of orofacial movements. On the other hand, Lee et al. (2010) found decreases in high-frequency power $(30-80 \mathrm{~Hz})$ in bilateral STS in response to viewing dynamic face stimuli portraying rigid motion, such as head turns that conveyed shifts in social attention. Interestingly, they did not find decreases in low-frequency 
power $(5-30 \mathrm{~Hz})$ in the STS. They did, however, report low-frequency power $(5-30 \mathrm{~Hz})$ decreases in the fusiform gyrus, in response to both dynamic and static face stimuli, which were greater in magnitude and spatial extent for the dynamic face stimuli. The authors interpreted these oscillatory power decreases in both low- and highfrequency bands as representing increases in cortical activation, in both ventral and dorsal streams during passive viewing of face stimuli.

A more recent MEG study by Jabbi et al. (2015) reported changes in sustained MEG beta-band (14-30 Hz) oscillatory activity during observation of dynamic faces, specifically happy and fearful facial displays, relative to their static counterparts in STS and frontolimbic cortices. However, they found that the modulation of oscillatory activity in response to facial dynamics was specific to the beta frequency band only, which they claim is consistent with the role of beta-band activity in visual and social information processing. They also report concordance with fMRI data where they found convergence of sustained BOLD signal and beta-band oscillatory responses in the STS for dynamic face stimuli. Likewise, Singh et al. (2002) compared the results from their MEG and SAM analysis with fMRI data acquired using the same paradigm and found that the fMRI BOLD response was inversely related to cortical synchronization within these lower-frequency (5-15 and 15-25 Hz) bands.

By combining their MEG and fMRI data while using the same paradigms, these studies provide a means to better link neurophysiological oscillatory responses to anatomical networks (Hall, Robson, Morris, \& Brookes, 2014). Moreover, there is a growing body of evidence suggesting that increased neural BOLD activation is represented by decreases in cortical oscillatory power in the low frequency range including alpha and beta frequencies (Hall et al., 2014; Zumer, Brookes, Stevenson, Francis, \& Morris, 2010). The current study therefore aims to explore neural responses to dynamic faces in the alpha and beta frequency ranges within the dynamic face perception network using a similar paradigm to that employed in a previous fMRI study (Foley et al., 2012). It is still unclear how modulations in low-frequency (8$30 \mathrm{~Hz}$ ) oscillatory brain activity within the distributed face perception network contribute to or reflect processing of dynamic facial displays and how these differ between dynamic and static displays. Hence, the overall aim of this study was to investigate the spatiotemporal and neural oscillatory responses to realistic dynamic face stimuli compared directly with their static counterparts. On the basis of the existing literature, our primary hypothesis is that dynamic face processing will be associated with modulations in alpha and beta power (8-30 Hz) within regions of the distributed face perception network including occipital, temporal, and frontal regions.

Specifically, we predict that changes in oscillatory power within alpha and beta bands will be larger for the dynamic face stimuli and will exhibit greater de- creases in power relative to their static counterparts. Furthermore, we expect that there will be overlap between the source locations of oscillatory power decreases identified in the SAM source analysis for the contrast of dynamic and static faces and the anatomical sources identified as showing increased BOLD responses for dynamic relative to static faces in our previous fMRI study (Foley et al., 2012). We further predict that responses in different regions of the distributed face perception network will display different temporal patterns of activation; specifically, sources within occipital gyri will display earlier peak responses for early visual analysis, followed by later responses in temporal and frontal regions, which are recruited later for cognitive and social processing (Adolphs, 2002a).

\section{METHODS \\ Participants}

Fourteen healthy, self-reported, right-handed volunteers (five men) with normal or corrected-to-normal vision (mean age $=29.2$ years, $S D=2.45$ years) gave full written informed consent to take part in the study, which was approved by the Aston University Human Science Ethical Committee.

\section{Experimental Design and Imaging Paradigm}

The paradigm was similar to that used in our previous study (see Foley et al., 2012, for a full description of stimulus creation and validation). The stimuli consisted of various dynamic and static angry, happy, and speech facial displays. For both static and dynamic conditions, images appeared on screen for $2.5 \mathrm{sec}$. The dynamic angry and happy video clips evolved over this period of $2.5 \mathrm{sec}$ from a closed-mouthed neutral expression to their respective emotional expression, reaching peak expression between approximately 1 and $1.5 \mathrm{sec}$. They then maintained peak expression for the remaining duration of the video clip. In the dynamic condition, four different identities were presented in each of the three different display categories (i.e., angry, happy, speech) and likewise in the static condition. The identities were matched across the dynamic and static conditions, as the static stimuli were created from a screenshot of the final frame of each of the dynamic excerpts.

A sample of 24 stimuli (12 dynamic and 12 corresponding static images) were presented in an event-related design, and participants were instructed to maintain central fixation throughout the experiment. Stimuli were completely randomized and presented for $2.5 \mathrm{sec}$, with $2.5 \mathrm{sec}$ of baseline (fixation cross) presented before each stimulus. There were 240 trials in an experimental run. Each trial consisted of a single stimulus presentation of $2.5 \mathrm{sec}$ (active state) and an ISI fixation cross of $2.5 \mathrm{sec}$ (passive state). Participants performed a 1-back memory 


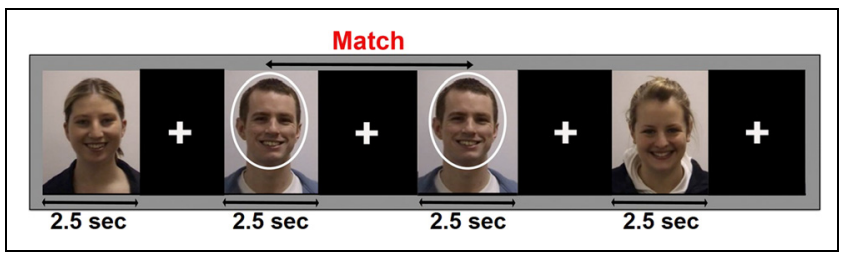

Figure 1. MEG experimental design: 120 dynamic and 120 static images were presented for $2.5 \mathrm{sec}$ in a random sequence, alternating with a 2.5-sec fixation cross. Participants $(N=14)$ performed a 1-back memory task and responded via button press as to whether the identity of the current image matched the previous image.

task and responded with their dominant hand via the lumina response pad as to whether the identity in the current image matched that of the previous image. They were asked to respond as soon as the stimulus appeared on screen. This is the same task that was used in the fMRI study, and as described in Foley et al. (2012), it was designed to maintain vigilance and to control for attention (see Figure 1).

MEG data were recorded using a 275-channel CTF system using third-order gradiometer configuration with a sampling rate of $600 \mathrm{~Hz}$. Three electromagnetic localization coils were attached to the participants' head at the nasion and bilateral preauricular points to localize their head relative to the MEG sensors. Continuous head motion was monitored throughout the recording, and all participants remained within the $1-\mathrm{cm}$ range to reduce contamination from motion artifacts. Participants were seated in an upright position in the MEG scanner. Visual stimuli were presented using Presentation (Neurobehavioral Systems, Inc., Berkeley, CA), and participants viewed the computer monitor directly through a window in the shielded room. A Polhemus Isotrak 3-D digitizer was used to map the surface shape of each participant's head and localize the electromagnetic head coils with respect to that surface. Each participant's head shape file was then extracted and coregistered to a high-resolution T1-weighted anatomical MRI, which was acquired for each participant on a 3-T Siemens Magnetom Trio Scanner using an eight-channel radio-frequency birdcage head coil. Coregistration was performed using in-house software based on an algorithm designed to minimize the squared Euclidean distance between the Polhemus surface and the MRI surface. This coregistration is accurate to within $5 \mathrm{~mm}$ (for further details, see Adjamian et al., 2004).

\section{Data Analysis}

Data for each participant were edited and filtered to remove environmental artifacts, a $50-\mathrm{Hz}$ powerline filter was used, and DC offset was removed. Third-order gradient noise reduction was also used to remove environmental noise from the data that was picked up by reference coils during acquisition. The MEG data were analyzed using SAM, which is a spatial filtering "beamformer" technique that can be used to generate SPMs of stimulus or event-related changes in cortical oscillatory power. A boxcar experimental design was used to assess spectral power between active (dynamic faces) and passive (static faces) states in alpha $(8-12 \mathrm{~Hz})$ and beta (13$30 \mathrm{~Hz}$ ) frequency bands. The difference between the active and passive spectral power estimates was assessed for each voxel using a pseudo $t$ statistic (Robinson \& Vrba, 1999). This produced a 3-D SAM image of cortical activity for each participant under each condition. SAM analysis was computed using 500-msec time windows to assess the main effect of motion by directly comparing power changes in low-frequency bands between dynamic and static faces, starting from stimulus onset at $0 \mathrm{msec}$ (0-500, 500-1000, 1000-1500, 1500-2000, and 2000$2500 \mathrm{msec}$ ). Rather than using a long time window of $2.5 \mathrm{sec}$ to cover the length of stimulus display, these 500-msec time windows were chosen to investigate the temporal progression within the network at multiple time points.

Each participant's data were normalized and converted to Talairach space using SPM (SPM99, www.fil.ion.ucl.ac. uk/spm) for group level comparisons. Nonparametric permutation analysis using SnPM (www.fil.ion.ucl.ac.uk/ spm) was computed to assess significant group effects. The output of the beamformer may be described as a "virtual electrode," which can be visualized as timefrequency plots of activity arising from specific voxels where spectral power changes are identified (Singh et al., 2002), and these can be used to characterize frequency-specific spectral power changes associated with the task in more detail (Hillebrand et al., 2005). ROIs were determined based on significant group effects identified with SnPM ( $p<.05$, corrected) as showing significant peaks of activation within the face perception network, and locations were verified against our previous fMRI study (Foley et al., 2012). These included bilateral inferior occipital gyri (IOGs), MTGs, and superior temporal gyri. To provide a more detailed estimation of the time-frequency characteristics of the signal within each ROI, virtual electrodes were constructed at the individual level at the sites of peak activation that fell within $1-\mathrm{cm}$ radius of the group peak identified with SnPM.

The virtual electrodes were based on a covariance matrix constructed using a 5 -sec window from $2.5 \mathrm{sec}$ before stimulus onset to $2.5 \mathrm{sec}$ after stimulus onset, with a bandwidth of 1-30 Hz. Time windows for baseline estimation were of equal duration to the time window of interest to achieve balanced covariance estimation. For visualization purposes, time-frequency wavelet plots were computed in MATLAB R2008b (The MathWorks, Natick, MA) on the virtual electrodes for a window beginning at $0-2.5 \mathrm{sec}$ after stimulus onset. Percent power change from baseline (the $1 \mathrm{sec}$ preceding stimulus onset) was computed at each frequency for both dynamic and static stimuli to give mean (across epochs and 
participants) power increases and decreases for dynamic and static face stimuli separately for each participant. Dynamic and static face stimuli were then directly contrasted at each ROI from 0 to $2.5 \mathrm{sec}$ after stimulus onset, across the group of participants, using a bootstrapping technique (Fisher et al., 2008; Singh et al., 2002). Only those changes that were significant at $p<.05$ are reported to correspond with the contrast performed at the wholehead group level.

An important aspect of this study is the direct comparison of two "active" states in the SAM analysis, rather than "active" versus "passive," as used in previous MEG and SAM studies (Lee et al., 2010; Muthukumaraswamy et al., 2006; Singh et al., 2002), hence in this study, dynamic faces were directly compared with static faces, not with baseline. This methodology was employed to use a more robust control for the dynamic face stimuli (Kaiser, Rahm, \& Lutzenberger, 2008) and to maintain consistency with our earlier fMRI study (Foley et al., 2012). However, to correctly interpret the results from the direct comparison of the dynamic versus static face conditions, the baseline comparisons must also be computed. This is because an overall decrease in the dynamic versus static face comparison may be driven by either a decrease in power for dynamic faces or an increase in power for static faces, hence time-frequency plots for the direct comparison along with the two baseline conditions were computed.

\section{RESULTS}

\section{Group Source Analysis Results}

SAM was computed across five different 500-msec time windows to identify sources of differential activity between dynamic and static face stimuli across the length of stimulus presentation in alpha and beta frequency bands. Group analysis was performed using SnPM to identify significantly clustered peaks across the group of participants in response to dynamic versus static face stimuli. As predicted, group SnPM analysis identified significant decreases in power in alpha and beta bands for dynamic relative to static faces, in regions within the distributed face perception network across the group of 14 participants (see Table 1 and Figure 2).

The first time window analyzed was from 0 to $500 \mathrm{msec}$ after stimulus onset, and this revealed an early decrease in low-frequency power in bilateral IOGs in response to dynamic relative to static faces. The later time window of 500-1000 msec again revealed decreases in low-frequency power in bilateral IOGs, along with bilateral MTGs, right STS, and left inferior frontal gyrus. Within the time window from 1000 to $1500 \mathrm{msec}$, decreases in lowfrequency power were seen in bilateral IOGs, right MTG, right STS, right lingual gyrus, right inferior frontal gyrus, and left insula. Notably, responses are more right lateralized during this period, and responses in the
Table 1. Brain Regions for Group SAM Analysis Showing Decreases in Alpha and Beta Power (8-30 Hz) for Dynamic Compared with Static Faces within the Following Time Windows: (A) 0-500 msec, (B) 500-1000 $\mathrm{msec}$, (C) 1000-1500 msec, (D) 1500-2000 msec, and (E) 2000-2500 msec

\begin{tabular}{|c|c|c|}
\hline & Pseudo $t$ Val & $x, y, z$ \\
\hline \multicolumn{3}{|l|}{ (A) $0-500 \mathrm{msec}$} \\
\hline L IOG (BA 17) & -3.6 & $-12,-93,-18$ \\
\hline R IOG (BA 18) & -3.24 & $18,-82,-11$ \\
\hline \multicolumn{3}{|l|}{ (B) 500-1000 msec } \\
\hline L MTG (BA 37) & -4.35 & $-42,-63,0$ \\
\hline R MTG (BA 39) & -3.83 & $48,-66,15$ \\
\hline L IOG (BA 18) & -3.54 & $-27,-99,-18$ \\
\hline R IOG (BA 18) & -3.47 & $27,-81,-9$ \\
\hline R STS (BA 22) & -2.8 & $54,-15,0$ \\
\hline L IOG (BA 18) & -6.03 & $-30,-81,-3$ \\
\hline R MTG (BA 37) & -5.97 & $42,-63,9$ \\
\hline R STS (BA 22) & -5.35 & $54,-15,0$ \\
\hline R IOG (BA 17) & -5.23 & $12,-87,-6$ \\
\hline L STS (BA 13) & -4.83 & $-51,-39,18$ \\
\hline $\mathrm{R}$ inferior frontal gyrus (BA 46) & -4.00 & $36,30,21$ \\
\hline L insula (BA 13) & -2.93 & $-36,21,12$ \\
\hline
\end{tabular}

\section{(D) $1500-2000 \mathrm{msec}$}

$\begin{array}{lrl}\text { R MTG (BA 19) } & -4.96 & 45,-63,12 \\ \text { R STS (BA 22) } & -4.50 & 57,-9,3 \\ \text { R IOG (BA 17) } & -4.10 & 12,-87,-6 \\ \text { L STS (BA 13) } & -3.74 & -51,-39,18 \\ \text { R middle occipital gyrus (BA 18) } & -3.32 & 15,-99,18 \\ \text { R inferior frontal gyrus (BA 47) } & -2.85 & 57,27,-6 \\ \text { R inferior frontal gyrus (BA 10) } & -2.33 & 42,39,12\end{array}$

(E) 2000-2500 $\mathrm{msec}$

R middle occipital gyrus (BA 18)

\begin{tabular}{lc}
-4.34 & $30,-84,-3$ \\
-3.15 & $60,-15,0$ \\
-3.11 & $-9,-24,68$ \\
-2.71 & $-45,-45,-60$ \\
-2.43 & $48,21,-30$ \\
-2.38 & $39,45,21$ \\
\hline
\end{tabular}

R STS (BA 22)

L precentral gyrus (BA 6)

L MTG (BA 37)

R STS (BA 38)

R middle frontal gyrus (BA 10)

Coordinates indicate local maxima in Talairach space. Clusters are significant at $p<.05 . \mathrm{L}=$ left; $\mathrm{R}=$ right. 
lingual gyrus, MTG, and STS show higher levels of activation as indexed by the higher $t$ values. Interestingly, right STS shows a sustained response from approximately 500 to 1500 msec.

From 1500 to $2000 \mathrm{msec}$, low-frequency power decreases were localized to the right MTG, bilateral STSs, right IOG, right middle occipital gyrus, and right inferior frontal gyrus. Again, these responses appear to be more right lateralized. Finally, from 2000 to $2500 \mathrm{msec}$, lowfrequency power decreases were found in the right middle occipital gyrus, right STS, left MTG, left precentral gyrus, right middle frontal gyrus, and left inferior frontal gyrus (see Table 1 and Figure 2).

\section{Group Time-Frequency Results}

Virtual electrodes were constructed to map the timefrequency characteristics of the ROIs within the dynamic face perception network (see Table 2 for coordinates of the virtual electrodes). The specific ROIs selected were bilateral IOGs, bilateral MTGs, and bilateral STSs, as these were the most robust regions identified across the group

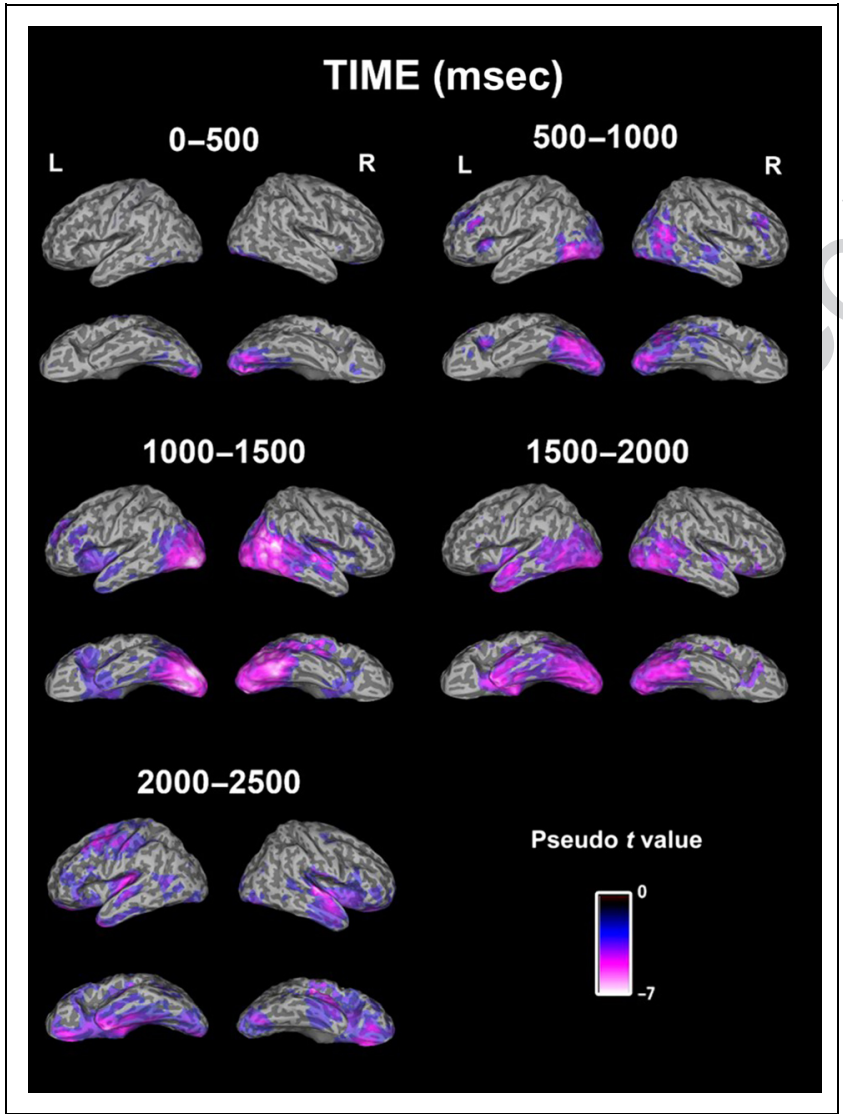

Figure 2. Group SAM image $(N=14)$ showing decreases in alpha and beta power for dynamic versus static faces within five different time windows: $0-500,500-1000,1000-1500,1500-2000$, and 2000-2500 msec after stimulus onset. The figure shows progression of activation within the face perception network over time. Activation is shown in bilateral IOGs, MTGs, superior temporal gyri, and inferior frontal gyri. Blue-purple-white color scale represents a decrease in signal power $(p<.05$, corrected).
Table 2. Mean Stereotactic Coordinates for the Virtual Electrodes in Talairach Space

\begin{tabular}{lcc}
\hline Region & $N$ & $x, y, z$ \\
\hline Left IOG & 12 & $-12,-93,-18$ \\
Right IOG & 12 & $20 .-81,-11$ \\
Left MTG & 8 & $-45,-45,-60$ \\
Right MTG & 8 & $42,-63,9$ \\
Left STS & 8 & $-51,-39,18$ \\
Right STS & 9 & $57,-9,3$ \\
\hline
\end{tabular}

$N=$ number of participants showing a significant peak in a particular region.

of participants in the SAM source analysis, which also corresponded to the regions within the core face perception network identified in our previous fMRI study (Foley et al., 2012). Virtual electrodes were constructed at the individual level only at sites of peak activation that fell within $1-\mathrm{cm}$ radius of the group peak identified with SnPM (see Table 2).

\section{IOGs}

Peak decreases in oscillatory power were identified in 12 of 14 participants in bilateral IOGs (see Table 2). The virtual electrodes constructed in the region in the left IOG (see Figure 3) showed, at the group level, a sustained power decrease within 200 msec of stimulus onset for dynamic faces relative to baseline in the $10-$ to $30-\mathrm{Hz}$ frequency range. Static faces compared with baseline showed a slight power increase at approximately $80 \mathrm{msec}$ between 20 and $30 \mathrm{~Hz}$, followed by a power decrease between 10 and $25 \mathrm{~Hz}$, which was not quite as sustained as the decrease to dynamic faces. The direct comparison of dynamic and static faces showed an early power decrease within the first 200 msec between 8 and $30 \mathrm{~Hz}$, followed by a slight increase and then a sustained decrease from approximately $700 \mathrm{msec}$ onward.

The time-frequency characteristics of the virtual electrodes constructed in the right IOG were extremely similar to those of the left IOG described above (see Figure 4). When dynamic faces were compared with baseline, a sustained power decrease within $200 \mathrm{msec}$ of stimulus onset was found in the 10- to $20-\mathrm{Hz}$ frequency range. Again, static faces compared with baseline showed a slight power increase around $80 \mathrm{msec}$ between 10 and $30 \mathrm{~Hz}$, followed by a power decrease around $200 \mathrm{msec}$ between 10 and $30 \mathrm{~Hz}$. When the response to dynamic and static faces was directly compared, an early power decrease within the first 200 msec between 10 and $30 \mathrm{~Hz}$ was found, followed by a later decrease around $800 \mathrm{msec}$ in the $10-$ to $30-\mathrm{Hz}$ range. 


\section{MTGs}

Peak decreases in oscillatory power were identified in 8 of 14 participants in bilateral MTGs (see Table 2). The virtual electrodes constructed in the left MTG (see Figure 5) revealed, at the group level, a sustained decrease in power from approximately $200 \mathrm{msec}$ in the $20-$ to $30-\mathrm{Hz}$ frequency range for dynamic faces relative to baseline, followed by a stronger sustained decrease in power from approximately $500 \mathrm{msec}$ between 10 and $15 \mathrm{~Hz}$. Static faces compared with baseline also showed a power decrease in the 20- to $30-\mathrm{Hz}$ frequency range, but this was at a slightly later time of $400 \mathrm{msec}$ and was not sustained. A similar decrease in power between 10 and $15 \mathrm{~Hz}$ was also revealed at $500 \mathrm{msec}$, but again, it was not as strong or sustained. When dynamic and static faces were directly compared, an early power decrease occurred at $200 \mathrm{msec}$ between 12 and $25 \mathrm{~Hz}$, followed by a decrease in power between 800 and $1500 \mathrm{msec}$ in the 8- to 30-Hz frequency range.

In the right MTG (see Figure 6), dynamic faces compared with baseline showed a sustained decrease in power from approximately $500 \mathrm{msec}$ between 10 and $30 \mathrm{~Hz}$. Static faces compared with baseline revealed a decrease in power between 400 and $800 \mathrm{msec}$ in the 10- to $30-\mathrm{Hz}$ range, followed by a later decrease in power from $1600 \mathrm{msec}$ between 8 and $25 \mathrm{~Hz}$. The direct contrast of dynamic and static faces showed a power increase from 400 to $800 \mathrm{msec}$ between 10 and $20 \mathrm{~Hz}$, which was driven by the corresponding decrease in power at this time for the static faces. This was followed by a decrease in power between 900 and $2000 \mathrm{msec}$ in the beta frequency range $(12-30 \mathrm{~Hz})$ due to the more sustained decrease in power for dynamic faces.

\section{STSs}

Peak decreases in oscillatory power were identified in 8 of 14 participants in the left superior temporal gyrus (see Table 2). When virtual electrodes were constructed in the left STS (see Figure 7), the contrast of dynamic faces with baseline showed, at the group level, a sustained decrease in power from $600 \mathrm{msec}$ onward around $12 \mathrm{~Hz}$. The static faces elicited a power decrease between 200 and $600 \mathrm{msec}$ in the $10-$ to $30-\mathrm{Hz}$ range. The direct comparison of dynamic and static faces showed a short power increase at $\sim 500 \mathrm{msec}$, followed by a stronger and more
Figure 3. Group $(N=12)$ time-frequency findings in left IOG (LIOG) show a significant early decrease in low-frequency power $(p<.05)$. (A) Dynamic faces compared with baseline show a sustained power decrease within $200 \mathrm{msec}$ of stimulus onset between 10 and $30 \mathrm{~Hz}$. (B) Static faces compared with baseline show a power increase at $80 \mathrm{msec}$, followed by a power decrease between 10 and $25 \mathrm{~Hz}$. (C) Direct comparison of dynamic and static faces shows an early power decrease within $200 \mathrm{msec}$ between 8 and $30 \mathrm{~Hz}$, followed by a sustained power decrease from $700 \mathrm{msec}$ onward. Black box indicates time windows showing significant differences between dynamic and static conditions that were identified in the whole-head group SAM analysis.

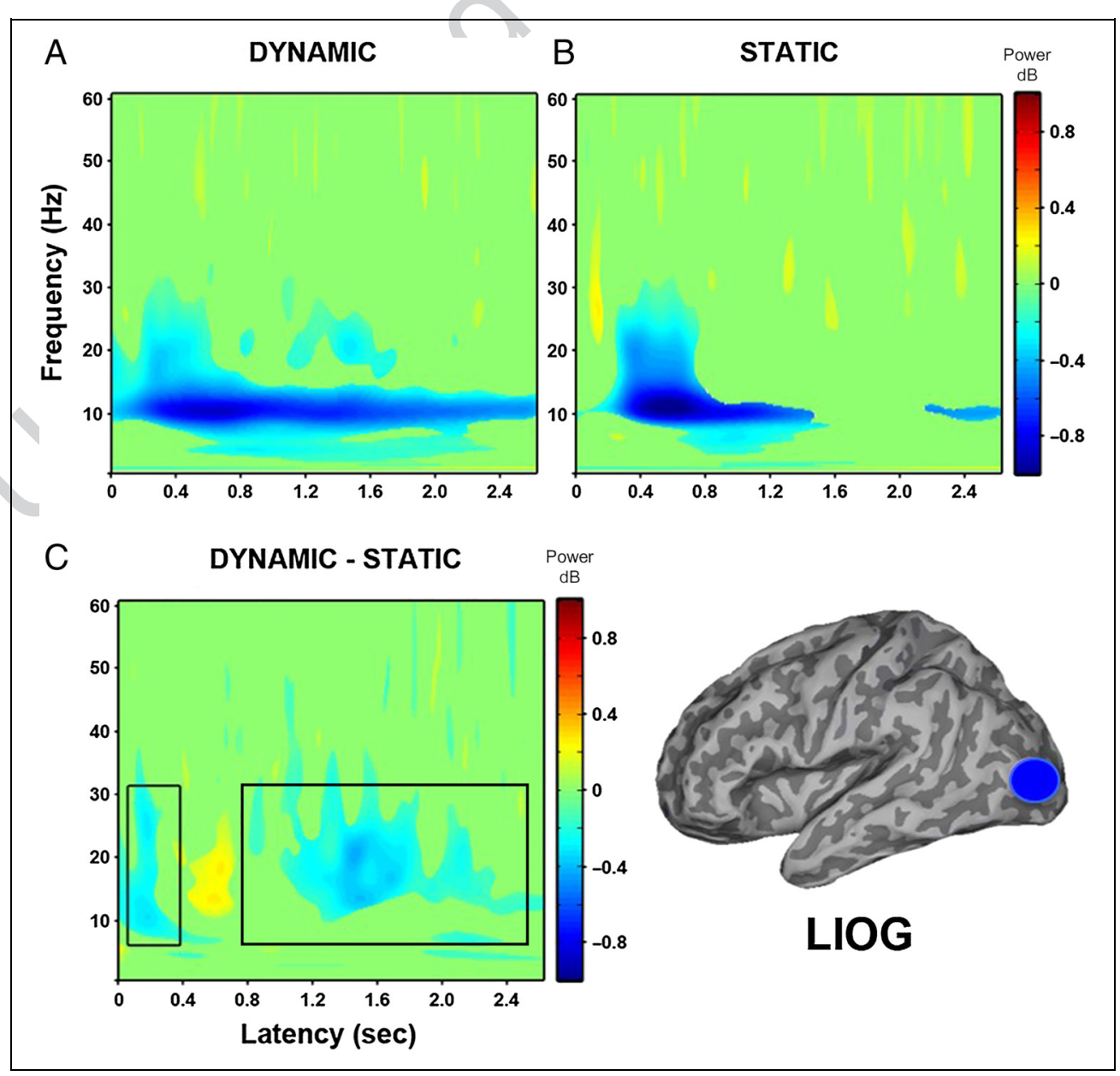


Figure 4. Group $(N=12)$ time-frequency findings in right IOG (RIOG) show a significant early decrease in low-frequency power $(p<.05)$. (A) Dynamic faces compared with baseline show a sustained power decrease within $200 \mathrm{msec}$ of stimulus onset between 10 and $30 \mathrm{~Hz}$. (B) Static faces compared with baseline show a power increase at $80 \mathrm{msec}$, followed by a power decrease between 10 and $25 \mathrm{~Hz}$. (C) Direct comparison of dynamic and static faces shows an early power decrease within 200 msec between 8 and $30 \mathrm{~Hz}$. Black box indicates time windows showing significant differences between dynamic and static conditions that were identified in the whole-head group SAM analysis.

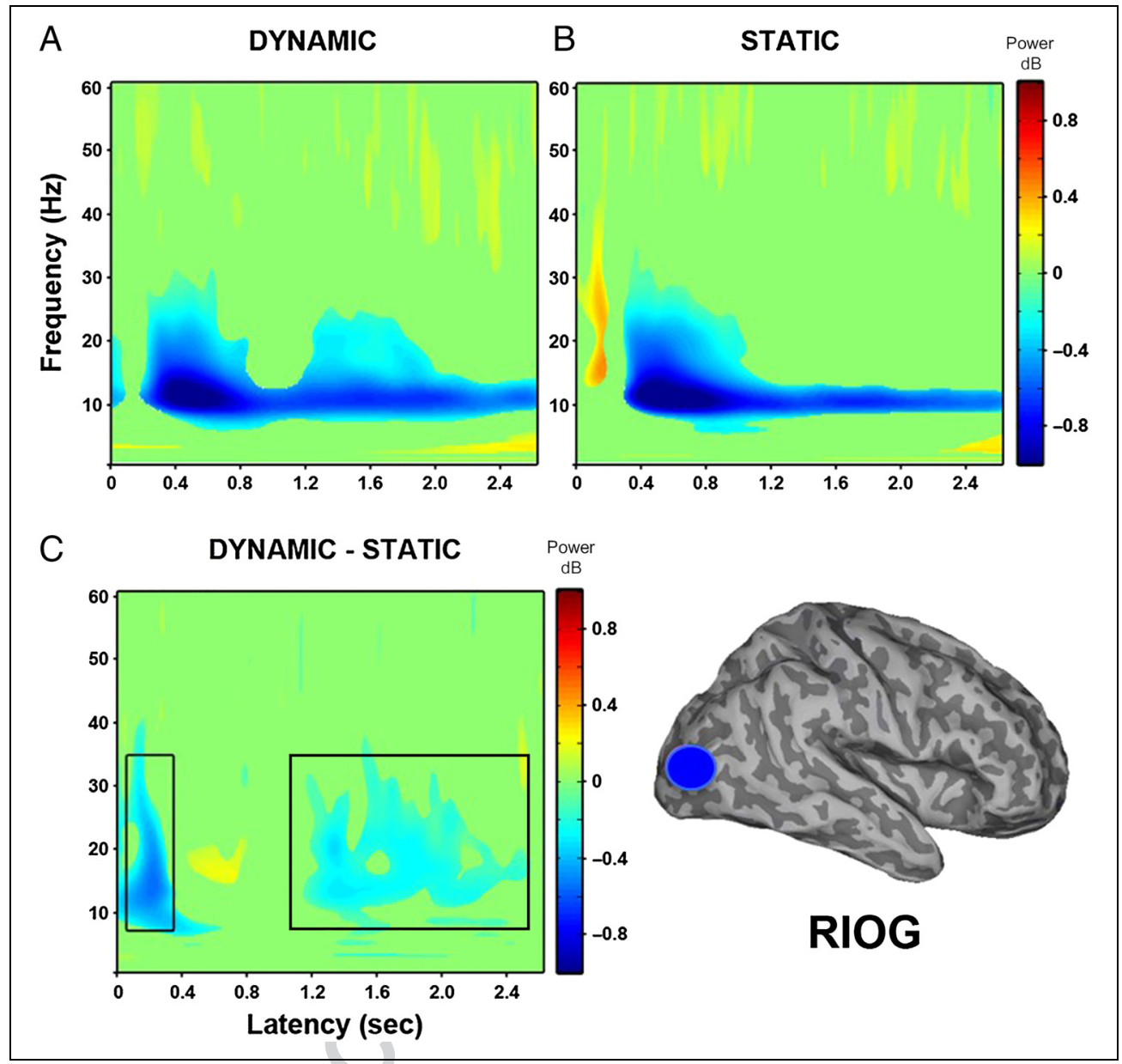

sustained decrease from $800 \mathrm{msec}$ onward between 10 and $30 \mathrm{~Hz}$ due to the more sustained decrease in power in the dynamic condition.

Peak decreases in oscillatory power were identified in 9 of 14 participants in the right superior temporal gyrus (see Table 2). The virtual electrodes constructed in the right STS (see Figure 8) revealed a broad decrease in power between 20 and $30 \mathrm{~Hz}$ from around 200 until $1600 \mathrm{msec}$ for the dynamic faces compared with baseline as well as a more sustained decrease in lower-frequency power $(8-15 \mathrm{~Hz})$ from $1000 \mathrm{msec}$ onward. Static faces compared with baseline showed a slight power decrease at $200 \mathrm{msec}$ and again at $600 \mathrm{msec}$ between 20 and $30 \mathrm{~Hz}$. Finally, the direct contrast of dynamic and static faces revealed a sustained decrease in power from $800 \mathrm{msec}$ onward between 8 and $30 \mathrm{~Hz}$ due to the decrease in power for dynamic faces.

\section{DISCUSSION}

This study sought to examine how changes in lowfrequency $(8-30 \mathrm{~Hz})$ oscillatory brain activity contribute to processing dynamic facial expressions by exploring how neural oscillatory profiles differ during perception of dynamic and static facial displays. To this end, MEG was used to explore the spatiotemporal and spectral power differences between dynamic and static face processing using realistic dynamic face stimuli. Whole-head beamformer source analyses revealed, at the millisecond level, significant differences in oscillatory power within alpha and beta frequency ranges $(8-30 \mathrm{~Hz}$ ) during processing of dynamic faces compared with their static counterparts in IC and occipito-temporal and frontal cortices. It was predicted that responses in these distinct regions of the distributed face perception network would display different temporal patterns of activation, whereby regions within occipital cortices would display earlier responses based on their role in early visual processing, followed by later responses in temporal and frontal cortices (Sato et al., 2015; Furl et al., 2010). This was confirmed through both the whole-head beamformer source analyses and the time-frequency analyses of virtual electrodes, which were constructed at regions of peak activation within the "core" face perception network.

Unlike many previous neurophysiologcal studies of dynamic face perception that have examined evoked 
activity (Miki \& Kakigi, 2014; Watanabe et al., 2005; Puce et al., 2000), we have focused our analyses on modulations of induced cortical oscillatory activity, which has been relatively less well studied in this context. Our motivation to focus on induced activity was guided by increasing evidence on the significant role of neural oscillations in cortical processing, where neural oscillations are now believed to play a key role in binding and information transfer between brain regions (Roopun et al., 2008; Buzsáki \& Draguhn, 2004; Engel et al., 2001). It is therefore important to examine information in the frequency domain in addition to the spatial and temporal domains, to gain a more thorough understanding of face processing in the brain. With a specific focus on dynamic face processing, there is evidence to suggest that perception of biological motion and facial dynamics are associated with modulations in alpha and beta frequency power in particular (Jabbi et al., 2015; Popov, Miller, Rockstroh, \& Weisz, 2013; Muthukumaraswamy et al., 2006; Singh et al., 2002). Hence, we concentrated our investigations on this range of frequencies $(8-30 \mathrm{~Hz})$ in this study.

The group SAM source analysis revealed a distributed network of brain regions showing differential responses for dynamic relative to static stimuli. This was characterized by greater decreases in low-frequency $(8-30 \mathrm{~Hz})$ power in response to viewing dynamic face stimuli. Early differential responses were identified in bilateral IOGs within $200 \mathrm{msec}$ of stimulus onset, followed by later responses in regions such as MTGs and STS within $800 \mathrm{msec}$ of stimulus onset. Additional regions within the so-called extended system, including the insula, inferior frontal and middle frontal gyri, and precentral gyri, all showed significantly greater decreases in low-frequency (8-30 Hz) oscillatory power for dynamic relative to static face stimuli from approximately $1000 \mathrm{msec}$ onward. The activation of this network of regions in response to dynamic face stimuli is consistent with findings from previous electrophysiological and neuroimaging studies (Sato et al., 2004, 2015; Foley et al., 2012). This provides converging evidence and additional insights into the role and neural signature of these regions during dynamic face perception. Furthermore, in this study, we endeavored to go beyond previous research on dynamic face processing by investigating oscillatory activity in more detail in specific ROIs. By computing virtual electrodes, it was possible to explore the profile of activity in specific regions within the face perception network and examine
Figure 5. Group $(N=8)$ timefrequency findings in left MTG (LMTG) show significant decreases in low-frequency power $(p<.05)$. (A) Dynamic faces compared with baseline show a sustained power decrease around $200 \mathrm{msec}$ between 20 and $30 \mathrm{~Hz}$ and a sustained power decrease around $500 \mathrm{msec}$ between 10 and $15 \mathrm{~Hz}$. (B) Static faces compared with baseline show a power decrease around 400 msec between 20 and 30 Hz. (C) Direct comparison of dynamic and static faces shows a power decrease around 200 msec between 12 and $25 \mathrm{~Hz}$ and a power decrease from 800 to 1500 msec between 8 and $30 \mathrm{~Hz}$. Black box indicates time windows showing significant differences between dynamic and static conditions that were identified in the whole-head group SAM analysis.

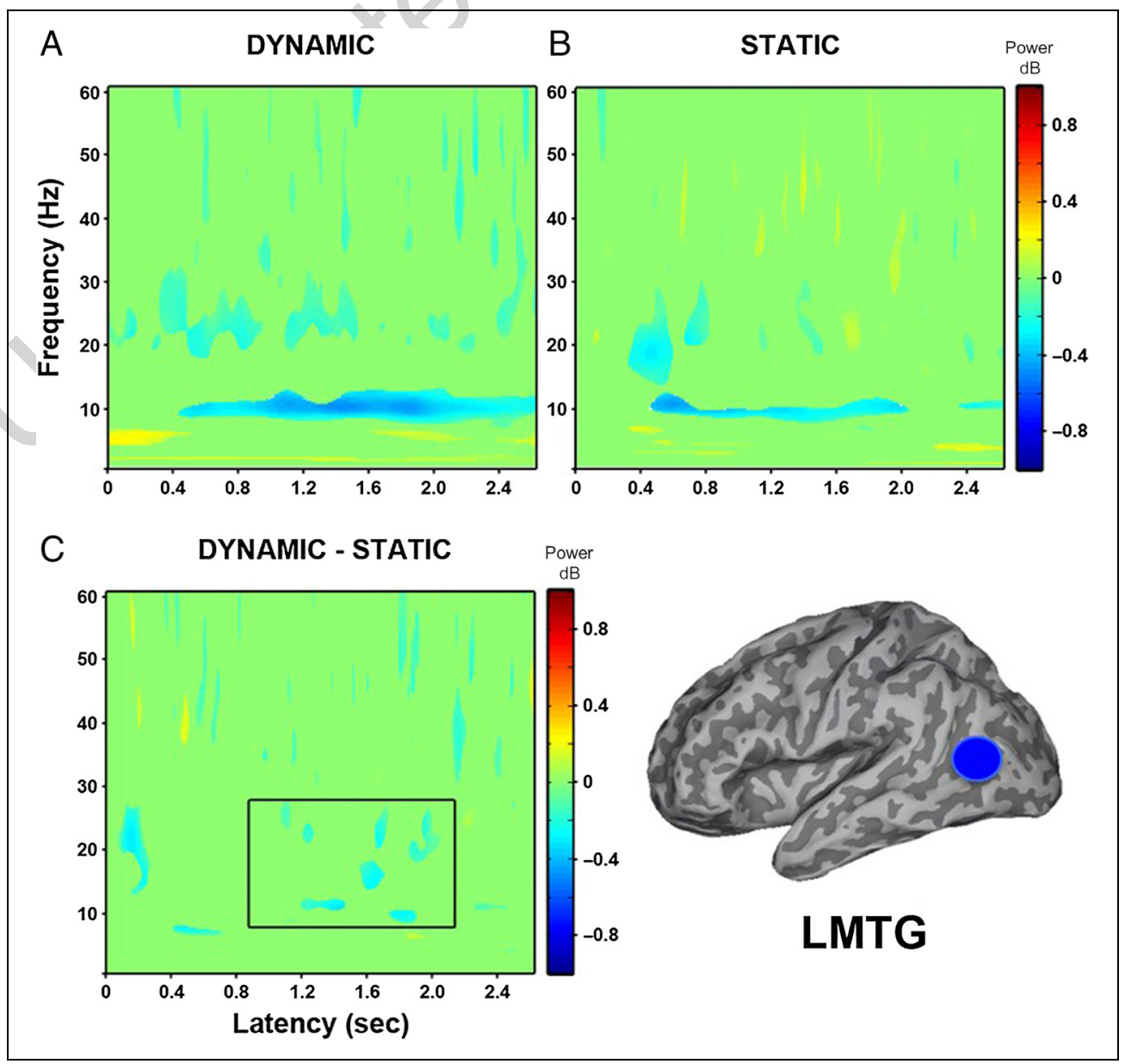


Figure 6. Group $(N=8)$ timefrequency findings in right MTG (RMTG) show significant decreases in low-frequency power $(p<.05)$. (A) Dynamic faces compared with baseline show a sustained power decrease around $500 \mathrm{msec}$ between 10 and $30 \mathrm{~Hz}$. (B) Static faces compared with baseline show a power decrease from 400 to 800 msec between 10 and $30 \mathrm{~Hz}$ and a later decrease around 1600 msec between 8 to $25 \mathrm{~Hz}$. (C) Direct comparison of dynamic and static faces shows a power increase from 400 to $800 \mathrm{msec}$ between 10 and $20 \mathrm{~Hz}$ and a power decrease around 900 msec between 12 and $30 \mathrm{~Hz}$. Black box indicates time windows showing significant differences between dynamic and static conditions that were identified in the whole-head group SAM analysis.

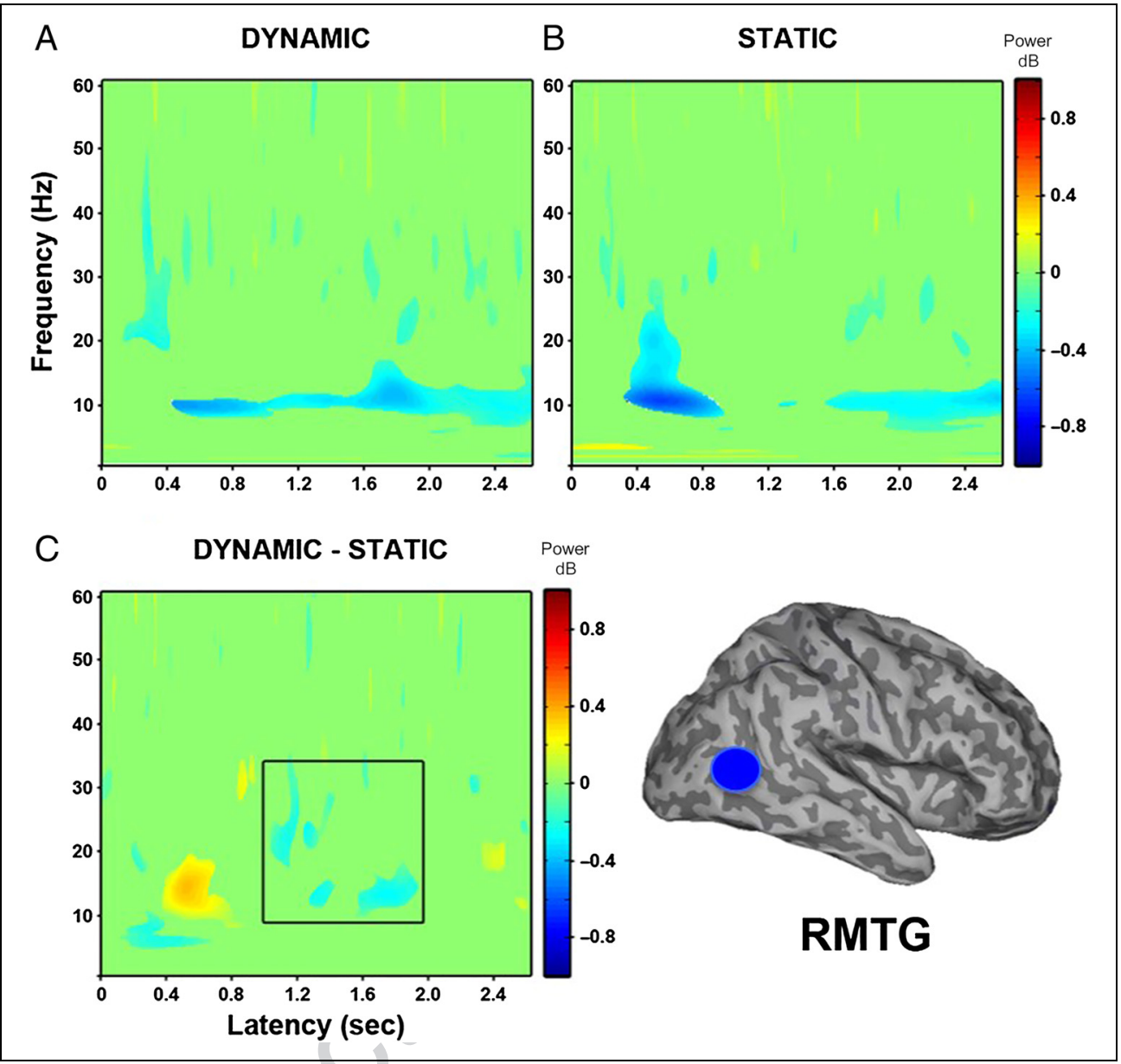

processing differences associated with dynamic and static faces in more detail.

The IOGs form part of the core system for early visual analysis of faces, as described in Haxby et al.'s (2000) model. Time-frequency analysis of the virtual SAM sensors that were constructed in bilateral IOG revealed differential patterns of activation for the dynamic and static face stimuli. There was a decrease in oscillatory power between 10 and $30 \mathrm{~Hz}$ in response to both the dynamic and static face stimuli within $200 \mathrm{msec}$ of stimulus onset, but overall, there was a significantly greater and more sustained decrease in power in the dynamic face condition. This early response in inferior occipital regions is consistent with evoked potential studies that have localized the major evoked response components (M100 and M170) that are known to occur during this period to occipital regions (Sato et al., 2015; Itier \& Taylor, 2002; Liu, Harris, \& Kanwisher, 2002). However, it must be noted that the current analysis method was designed to detect changes in oscillatory activity over time windows of hundreds of milliseconds and is relatively insensitive to more time-locked transient activity occurring over short intervals of time such as evoked responses.
The overall finding of significantly greater decreases in sustained alpha and beta power within bilateral IOG for dynamic relative to static faces suggests that the IOG is involved in processing both the static and dynamic face stimuli, but additional processing was required for the dynamic stimuli. This is consistent with fMRI data showing significant increases in BOLD activation in IOG for dynamic face stimuli (Fox et al., 2009; Schultz \& Pilz, 2009). The sustained response in IOG has important implications for hierarchical feedforward face perception models, as it suggests that IOG not only is involved in early visual analysis but also may play a role in higherlevel processing (Atkinson \& Adolphs, 2011) such as facial expression analysis (Pitcher, Garrido, Walsh, \& Duchaine, 2008), possibly due to afferent feedback connections from the STS (Furl, Henson, Friston, \& Calder, 2015; Foley et al., 2012).

Regions within bilateral MTGs, which include area MT, also showed significantly greater decreases in alpha and beta power for dynamic relative to static faces. Area MT is a well-known region in motion processing in both the fMRI and MEG literature (e.g., Sato et al., 2004; Singh et al., 2002; Ahlfors et al., 1999), and previous fMRI 
studies have implicated regions of MTGs in dynamic face processing (Sato et al., 2015; Foley et al., 2012; Trautmann et al., 2009). In an MEG study, Watanabe et al. (2005) identified area MT as a key region involved in facial motion processing. They examined evoked responses to facial movements and found that both eye and mouth movements elicited responses in this region around 170 msec after stimulus onset, with larger responses to eye movements. Area MT has also been shown to respond to biological motion between 100 and 200 msec of stimulus onset with EEG (Krakowski et al., 2011). In addition, Singh et al. (2002) reported a decrease in low-frequency power in MTGs when participants viewed point light displays of biological motion. Our results are consistent with previous findings showing that MTGs are involved in processing biological motion including dynamic facial displays, which appears to be facilitated through sustained decreases in oscillatory power between 8 and $30 \mathrm{~Hz}$.

The STS is another important structure within the face perception network that has consistently been shown to be involved in motion processing, including biological motion (Krakowski et al., 2011; Jokisch, Daum, Suchan, \& Troje, 2005; Singh et al., 2002), dynamic face processing (Furl et al., 2015; Jabbi et al., 2015; Foley et al., 2012;
Sato et al., 2004), and multimodal integration (Hagan, Woods, Johnson, Green, \& Young, 2013). Here, we found significant decreases in oscillatory power $(8-30 \mathrm{~Hz})$ in bilateral STS during dynamic face processing. Timefrequency analysis of the virtual SAM sensors constructed within regions of posterior STS revealed similar response patterns in the left and right STS sources. This was characterized by a brief decrease in power at $~ 500 \mathrm{msec}$ in both the static and dynamic conditions, which was then followed by a significantly stronger sustained decrease in power from $800 \mathrm{msec}$ onward in the dynamic condition only. It would therefore appear then that the STS contributes largely to the processing of dynamic faces and, to a lesser extent, static faces. This is consistent with results from neuroimaging fMRI studies, wherein the STS showed significantly greater activation for dynamic relative to static faces (Foley et al., 2012; Trautmann et al., 2009; Sato et al., 2004). In line with this, an fMRI study by Pitcher et al. (2011) reported a region in the right posterior STS that responded almost three times more strongly to dynamic compared with static faces. Increases in theta power can also be seen in the time-frequency plots in bilateral STS. We speculate that this may represent cross-frequency power coupling between theta and alpha/ beta frequency ranges during face processing, consistent
Figure 7. Group $(N=8)$ timefrequency findings in left STS (LSTS) show significant decreases in low-frequency power $(p<.05)$. (A) Dynamic faces compared with baseline show a sustained power decrease from 400 to $2000 \mathrm{msec}$ around $12 \mathrm{~Hz}$. (B) Static faces compared with baseline show a power decrease from 200 to 600 msec between 10 and $30 \mathrm{~Hz}$. (C) Direct comparison of dynamic and static faces shows significant sustained decreases in power from $800 \mathrm{msec}$ onward between 10 and $30 \mathrm{~Hz}$. Black box indicates time windows showing significant differences between dynamic and static conditions that were identified in the whole-head group SAM analysis.

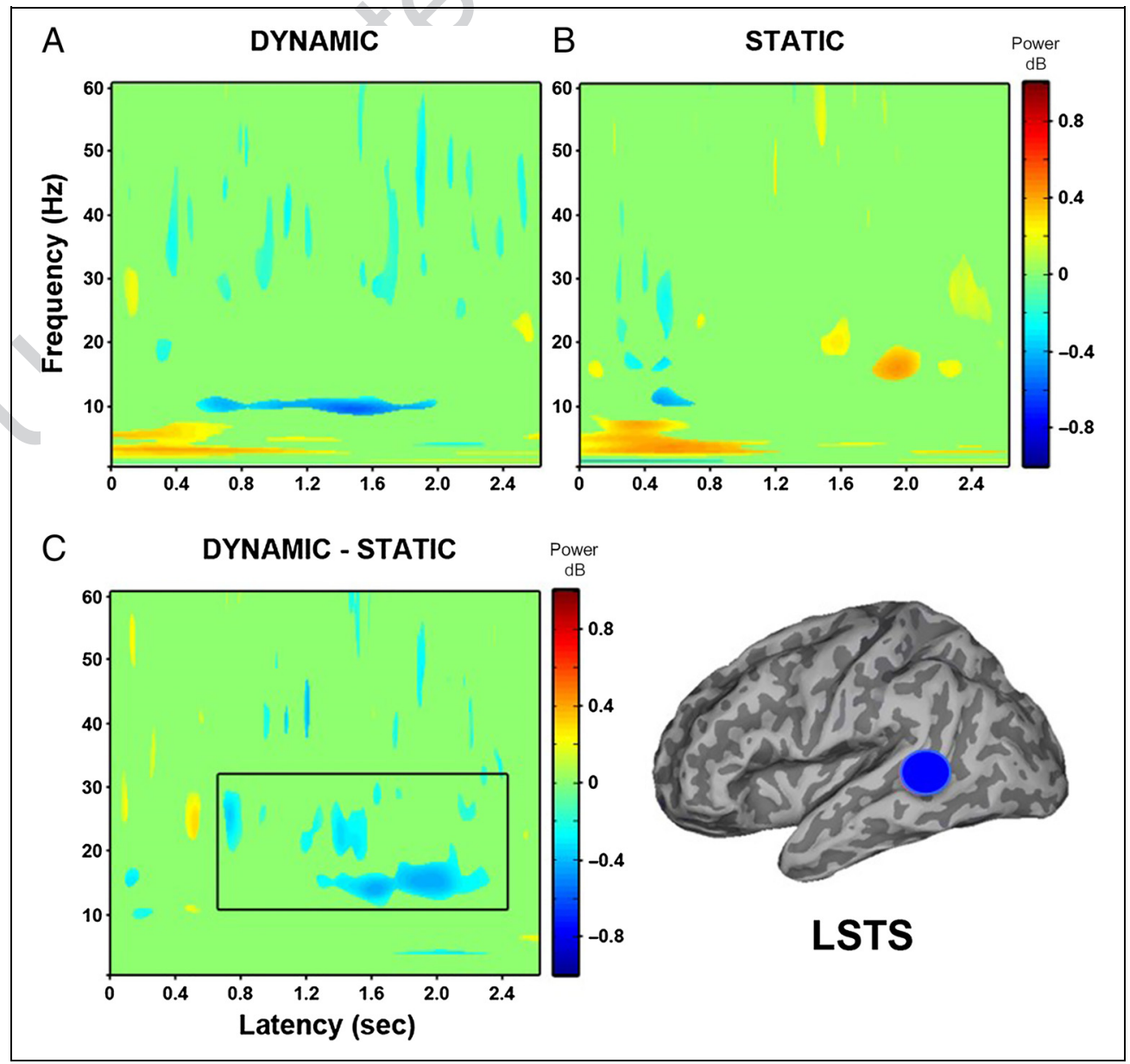


Figure 8. Group $(N=9)$ timefrequency findings in right STS (RSTS) show significant decreases in low-frequency power $(p<.05)$. (A) Dynamic faces compared with baseline show a power decrease between 20 and $30 \mathrm{~Hz}$ from 200 to 1600 msec and a sustained decrease in power between 8 and $15 \mathrm{~Hz}$ around 1000 msec. (B) Static faces compared with baseline show a power decrease between 20 and $30 \mathrm{~Hz}$ at 200 and 600 msec. (C) Direct comparison of dynamic and static faces shows significant sustained decreases in power from 800 msec onward between 8 and $30 \mathrm{~Hz}$. Black box indicates time windows showing significant differences between dynamic and static conditions that were identified in the whole-head group SAM analysis.

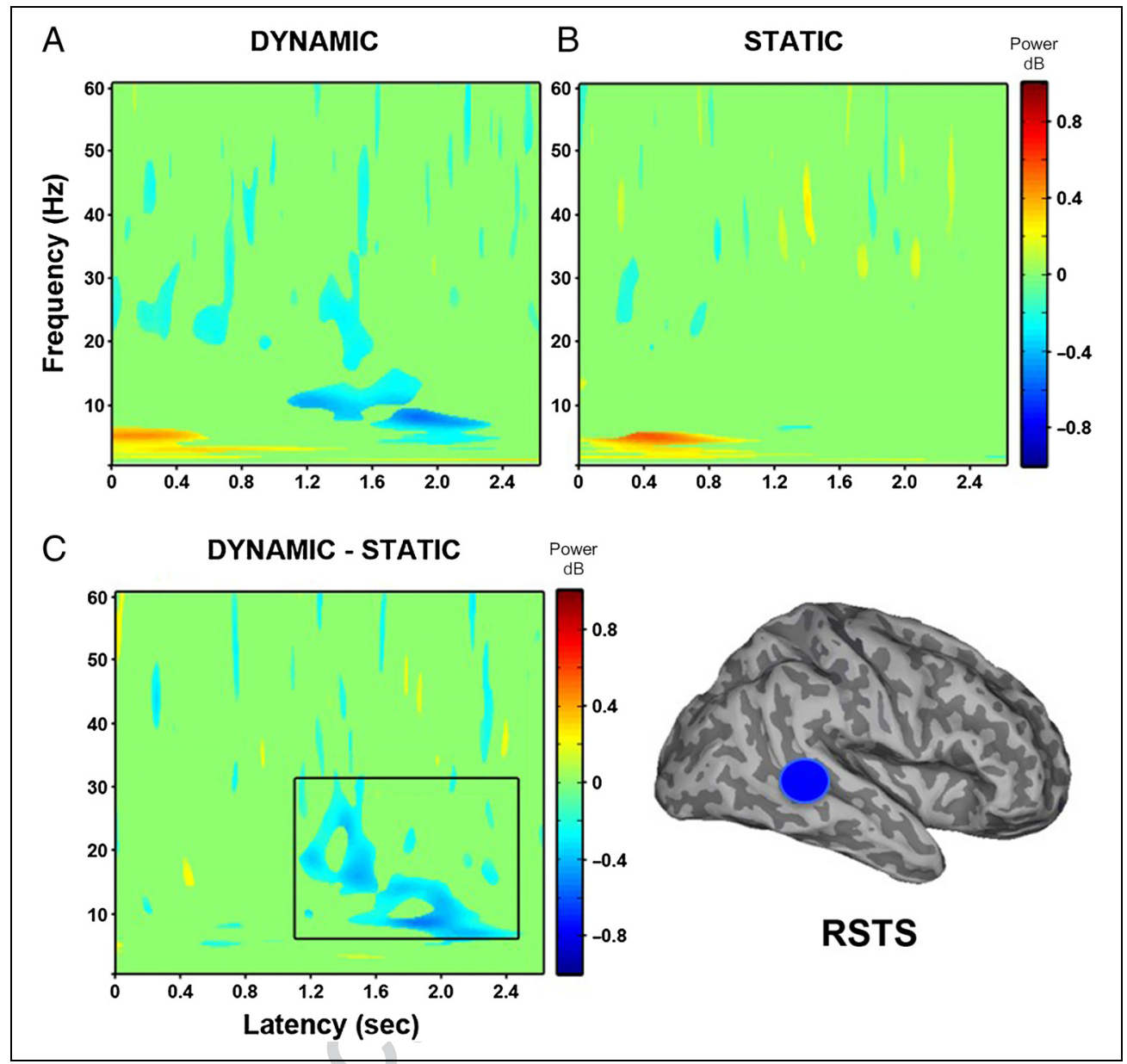

with recent findings from Furl et al. (2014) using static face stimuli. However, as it was not the focus of this study, a more specific analysis would be required to investigate the effects of cross-frequency power coupling during naturalistic dynamic face processing.

The STS possesses strong reciprocal connections with frontal and paralimbic regions as well as with visual cortical areas (Hein \& Knight, 2008). This unique arrangement of connections allows the STS to act as a functional interface between regions involved in early visual perceptual processing and emotion processing (Hein \& Knight, 2008). It has been proposed that modulations of oscillatory beta power in the STS associated with dynamic face processing may represent a mechanism to facilitate the integration of information from multiple input areas to attribute meaning to facial movements (Jabbi et al., 2015). In line with this, it has been shown that occipital visual regions accumulate information from dynamic face stimuli over shorter time intervals and thus respond faster (165 msec), whereas the STS accumulates information over longer time intervals resulting in a later response (237 msec), thereby enabling the integration of information from multiple sites (Furl et al., 2010). This is consistent with the pattern of results observed in this study, showing earlier decreases in oscillatory power $(8-30 \mathrm{~Hz})$ in inferior temporal gyrus and MTG, followed by later modulations in oscillatory activity in STS in response to dynamic facial displays.

Regions of the so-called extended face perception system, including sources in inferior and middle frontal gyri, left insula, and precentral gyrus, all showed significant decreases in oscillatory power between 8 and $30 \mathrm{~Hz}$ in response to dynamic relative to static face stimuli. These regions were all identified as structures forming part of the dynamic face perception network in our previous fMRI study, where they showed significant increases in BOLD signal in response to dynamic facial displays, demonstrating cross-modal convergence. Inferior frontal gyri, in particular, have been implicated in the perception of dynamic faces in both fMRI and MEG studies (Sato et al., 2004, 2015; Foley et al., 2012; Fox et al., 2009; Schultz \& Pilz, 2009). Inferior frontal regions are generally associated with top-down cognitive processes, hence the decreases in oscillatory power in inferior frontal sources identified in this study may reflect a form of additional top-down cognitive processing that is required for dynamic faces. This may be due to the fact that dynamic faces contain more information to be coordinated compared with static faces (Arsalidou, Morris, \& Taylor, 2011). The insula is believed to play an important role 
in emotion perception through its projections to the inferior pFC and amygdala (Phelps et al., 2001). It has also been associated with empathy (Adolphs, 2009) and language processing (for a review, see Oh, Duerden, \& Pang, 2014). Consistent with our findings, Jabbi et al. (2015) also reported decreased beta-band power in the left insula for dynamic face stimuli. This increased response for dynamic facial displays may be due to the increased salience of the dynamic face stimuli (Trautmann et al., 2009).

In summary, we found that, in comparison with static pictures of faces, dynamic images of faces were associated with significantly greater modulations in alpha and beta oscillatory activity across a distributed network of regions. Notably, all of the identified sources corresponded very closely with regions of the dynamic face perception network that were identified with fMRI in a preceding study using similar experimental protocols. These findings demonstrate strong concordance between two different imaging techniques and are consistent with a growing body of literature showing that the BOLD signal correlates negatively with alpha and beta oscillatory activity (Hall et al., 2014; Zumer et al., 2010; Singh et al., 2002) and adds support to the role of these signals in processing social stimuli (Engel \& Fries, 2010). Our results therefore demonstrate that perception of realistic dynamic facial stimuli activates a distributed neural network at varying time points facilitated by modulations in low-frequency power within alpha and beta frequency ranges (8-30 Hz).

An important aspect of this study that may have influenced the results is that two "active" states were compared in the SAM analysis rather than "active" versus "passive"; that is, dynamic faces were compared directly with static faces, not with baseline. In most studies using SAM analysis, an "active" state is compared with a "passive" state, generally represented by a prestimulus baseline period (Lee et al., 2010; Muthukumaraswamy et al., 2006; Singh et al., 2002). However, in this MEG study, a direct comparison between two active states was made between the dynamic and static face stimuli. This methodology was employed to use a more robust control for the dynamic face stimuli and to maintain consistency with our previous fMRI paradigm (Foley et al., 2012). Direct contrasts may yield more focal oscillatory activations than comparing prestimulus versus poststimulus responses (Kaiser et al., 2008). Hence, the patterns of activation described here may be more focal than those found in previous studies.

A limitation of this study that should be addressed in future research is the use of the 1-back identity recognition task, which may have biased face processing. This task was employed to ensure that participants maintained vigilance throughout and to facilitate comparison with our preceding fMRI study. However, the task may have influenced modulation in alpha activity in particular, as alpha oscillations are believed to play a role in optimizing performance in task-relevant regions and reducing processing in task-irrelevant regions (Jensen \& Mazaheri, 2010). This could be addressed in future studies by using task-free paradigms such as passive viewing of dynamic faces. It must also be noted that our task involved implicit recognition of facial expressions only and therefore the spatiotemporal and spectral profiles associated with explicit face processing should be addressed in future work. In addition, we did not include a control for nonbiological or nonfacial motion in this study. Although a contrast with nonfacial motion could provide information on facial-specific dynamics, we were specifically interested in exploring how neural oscillatory profiles differ during perception of dynamic as opposed to static facial displays. In line with Jabbi et al. (2015), our fMRI (Foley et al., 2012) and MEG analyses have elucidated the areas and mechanisms that are involved in biological facial motion while controlling for static information.

In conclusion, ecological validity is an important aspect of experimental research. Neuroscience research in general, but particularly, vision and face perception research, is striving toward the use of more naturalistic and ecologically valid stimuli and experimental designs (Johnston et al., 2015; Hasson, Malach, \& Heeger, 2010). This is exemplified by the growing trend of studying neural activation under more natural viewing conditions, such as while watching movies (see Hasson et al., 2010, for a review). This level of research is necessary as it aims to establish the functional significance of neural responses in natural conditions, which may have only been characterized with artificial stimuli (Felsen \& Dan, 2005). In this context, naturalistic dynamic face stimuli provide a better means of representing the complex nature of perceiving emotions from facial expressions in the real world. Therefore, authentic dynamic stimuli should be used to uncover the neural correlates of natural face perception, where the ultimate goal is to progress from an understanding of how static images of single faces are processed to how real faces are perceived dynamically and interactively in the real world (Atkinson \& Adolphs, 2011).

Reprint requests should be sent to Elaine Foley, Aston Brain Centre, School of Life and Health Sciences, Aston University, Birmingham B4 7ET, UK, or via e-mail: e.foley@aston.ac.uk.

\section{REFERENCES}

Adjamian, P., Barnes, G. R., Hillebrand, A., Holliday, I. E., Singh, K. D., Furlong, P. L., et al. (2004). Co-registration of magnetoencephalography with magnetic resonance imaging using bite-bar-based fiducials and surface-matching. Clinical Neurophysiology, 115, 691-698.

Adolphs, R. (2002a). Neural systems for recognizing emotion. Current Opinion in Neurobiology, 12, 169-177.

Adolphs, R. (2002b). Recognizing emotion from facial expressions: Psychological and neurological mechanisms. Behavioral and Cognitive Neuroscience Reviews, 1, 21-62. 
Adolphs, R. (2009). The social brain: Neural basis of social knowledge. Annual Review of Psychology, 60, 693-716.

Ahlfors, S. P., Simpson, G. V., Dale, A. M., Belliveau, J. W., Liu, A. K., Korvenoja, A., et al. (1999). Spatiotemporal activity of a cortical network for processing visual motion revealed by MEG and fMRI. Journal of Neurophysiology, 82, 2545-2555.

Ambadar, Z., Schooler, J. W., \& Cohn, J. F. (2005). Deciphering the enigmatic face: The importance of facial dynamics in interpreting subtle facial expressions. Psychological Science, 16, 403-410.

Arsalidou, M., Morris, D., \& Taylor, M. J. (2011). Converging evidence for the advantage of dynamic facial expressions. Brain Topography, 24, 149-163.

Atkinson, A. P., \& Adolphs, R. (2011). The neuropsychology of face perception: Beyond simple dissociations and functional selectivity. Philosophical Transactions of the Royal Society of London, Series B: Biological Sciences, 366, 1726-1738.

Buzsáki, G., \& Draguhn, A. (2004). Neuronal oscillations in cortical networks. Science, 304, 1926-1929.

Calder, A. J., \& Young, A. W. (2005). Understanding the recognition of facial identity and facial expression. Nature Reviews Neuroscience, 6, 641-651.

Donner, T. H., \& Siegel, M. (2011). A framework for local cortical oscillation patterns. Trends in Cognitive Sciences, 15, 191-199.

Engel, A. K., \& Fries, P. (2010). Beta-band oscillationsSignalling the status quo? Current Opinion in Neurobiology, 20, 156-165.

Engel, A. K., Fries, P., \& Singer, W. (2001). Dynamic predictions: Oscillations and synchrony in top-down processing. Nature Reviews Neuroscience, 2, 704-716.

Felsen, G., \& Dan, Y. (2005). A natural approach to studying vision. Nature Neuroscience, 8, 1643-1646.

Fisher, A. E., Furlong, P. L., Seri, S., Adjamian, P., Witton, C., Baldeweg, T., et al. (2008). Interhemispheric differences of spectral power in expressive language: A MEG study with clinical applications. International Journal of Psychophysiology, 68, 111-122.

Foley, E., Rippon, G., Thai, N. J., Longe, O., \& Senior, C. (2012). Dynamic facial expressions evoke distinct activation in the face perception network: A connectivity analysis study. Journal of Cognitive Neuroscience, 24, 507-520.

Fox, C. J., Iaria, G., \& Barton, J. J. S. (2009). Defining the face processing network: Optimization of the functional localizer in fMRI. Human Brain Mapping, 30, 1637-1651.

Furl, N., Coppola, R., Averbeck, B. B., \& Weinberger, D. R. (2014). Cross-frequency power coupling between hierarchically organized face-selective areas. Cerebral Cortex, 24, 2409-2420.

Furl, N., Henson, R. N., Friston, K. J., \& Calder, A. J. (2015). Network interactions explain sensitivity to dynamic faces in the superior temporal sulcus. Cerebral Cortex, 25, 2876-2882.

Furl, N., van Rijsbergen, N. J., Kiebel, S. J., Friston, K. J., Treves, A., \& Dolan, R. J. (2010). Modulation of perception and brain activity by predictable trajectories of facial expressions. Cerebral Cortex, 20, 694-703.

Hagan, C. C., Woods, W., Johnson, S., Green, G. G. R., \& Young, A. W. (2013). Involvement of right STS in audio-visual integration for affective speech demonstrated using MEG. PLoS One, 8, e70648.

Hall, E. L., Robson, S. E., Morris, P. G., \& Brookes, M. J. (2014). The relationship between MEG and fMRI. Neuroimage, 102, 80-91.

Hasson, U., Malach, R., \& Heeger, D. J. (2010). Reliability of cortical activity during natural stimulation. Trends in Cognitive Sciences, 14, 40-48.

Haxby, J. V., Hoffman, E. A., \& Gobbini, M. I. (2000). The distributed human neural system for face perception. Trends in Cognitive Sciences, 4, 223-233.
Haxby, J. V., Hoffman, E. A., \& Gobbini, M. I. (2002). Human neural systems for face recognition and social communication. Biological Psychiatry, 51, 59-67.

Hein, G., \& Knight, R. T. (2008). Superior temporal sulcus-It's my area: Or is it? Journal of Cognitive Neuroscience, 20, 2125-2136.

Hillebrand, A., Singh, K. D., Holliday, I. E., Furlong, P. L., \& Barnes, G. R. (2005). A new approach to neuroimaging with magnetoencephalography. Human Brain Mapping, 25, $199-211$

Ishai, A. (2008). Let's face it: It's a cortical network. Neuroimage, 40, 415-419.

Itier, R. J., \& Taylor, M. J. (2002). Inversion and contrast polarity reversal affect both encoding and recognition processes of unfamiliar faces: A repetition study using ERPs. Neuroimage, 15, 353-372.

Jabbi, M., Kohn, P. D., Nash, T., Ianni, A., Coutlee, C., Holroyd, T., et al. (2015). Convergent BOLD and beta-band activity in superior temporal sulcus and frontolimbic circuitry underpins human emotion cognition. Cerebral Cortex, 25, 1878-1888.

Jensen, O., \& Mazaheri, A. (2010). Shaping functional architecture by oscillatory alpha activity: Gating by inhibition. Frontiers in Human Neuroscience, 4, 186.

Johnston, P., Molyneux, R., \& Young, A. W. (2015). The N170 observed "in the wild": Robust event-related potentials to faces in cluttered dynamic visual scenes. Social Cognitive and Affective Neuroscience, 10, 938-944.

Jokisch, D., Daum, I., Suchan, B., \& Troje, N. F. (2005). Structural encoding and recognition of biological motion: Evidence from event-related potentials and source analysis. Behavioural Brain Research, 157, 195-204.

Kaiser, J., Rahm, B., \& Lutzenberger, W. (2008). Direct contrasts between experimental conditions may yield more focal oscillatory activations than comparing pre- versus poststimulus responses. Brain Research, 1235, 63-73.

Kilts, C. D., Egan, G., Gideon, D. A., Ely, T. D., \& Hoffman, J. M. (2003). Dissociable neural pathways are involved in the recognition of emotion in static and dynamic facial expressions. Neuroimage, 18, 156-168.

Krakowski, A. I., Ross, L. A., Snyder, A. C., Sehatpour, P., Kelly, S. P., \& Foxe, J. J. (2011). The neurophysiology of human biological motion processing: A high-density electrical mapping study. Neuroimage, 56, 373-383.

LaBar, K. S., Crupain, M. J., Voyvodic, J. T., \& McCarthy, G. (2003). Dynamic perception of facial affect and identity in the human brain. Cerebral Cortex, 13, 1023-1033.

Lee, L. C., Andrews, T. J., Johnson, S. J., Woods, W., Gouws, A., Green, G. G. R., et al. (2010). Neural responses to rigidly moving faces displaying shifts in social attention investigated with fMRI and MEG. Neuropsychologia, 48, 477-490.

Liu, J., Harris, A., \& Kanwisher, N. (2002). Stages of processing in face perception: An MEG study. Nature Neuroscience, 5, 910-916

Miki, K., \& Kakigi, R. (2014). Magnetoencephalographic study on facial movements. Frontiers in Human Neuroscience, $8,550$.

Muthukumaraswamy, S. D., Johnson, B. W., Gaetz, W. C., \& Cheyne, D. O. (2006). Neural processing of observed oro-facial movements reflects multiple action encoding strategies in the human brain. Brain Research, 1071, 105-112.

Oh, A., Duerden, E. G., \& Pang, E. W. (2014). The role of the insula in speech and language processing. Brain and Language, 135, 96-103.

Phelps, E. A., O’Connor, K. J., Gatenby, J. C., Gore, J. C., Grillon, C., \& Davis, M. (2001). Activation of the left amygdala to a cognitive representation of fear. Nature Neuroscience, 4 , 437-441. 
Pitcher, D., Dilks, D. D., Saxe, R. R., Triantafyllou, C., \& Kanwisher, N. (2011). Differential selectivity for dynamic versus static information in face-selective cortical regions. Neuroimage, 56, 2356-2363.

Pitcher, D., Garrido, L., Walsh, V., \& Duchaine, B. C. (2008). Transcranial magnetic stimulation disrupts the perception and embodiment of facial expressions. Journal of Neuroscience, 28, 8929-8933.

Popov, T., Miller, G. A., Rockstroh, B., \& Weisz, N. (2013). Modulation of $\alpha$ power and functional connectivity during facial affect recognition. Journal of Neuroscience, 33 , 6018-6026.

Puce, A., \& Perrett, D. (2003). Electrophysiology and brain imaging of biological motion. Philosophical Transactions of the Royal Society of London, Series B: Biological Sciences, 358, 435-445.

Puce, A., Smith, A., \& Allison, T. (2000). ERPs evoked by viewing facial movements. Cognitive Neuropsychology, 17, 221-239.

Robinson, S., \& Vrba, J. (1999). Functional neuroimaging by synthetic aperture magnetometry (SAM). In Recent advances in biomagnetism (pp. 302-305). Sendai, Japan: Tohoku University Press.

Roopun, A. K., Kramer, M. A., Carracedo, L. M., Kaiser, M., Davies, C. H., Traub, R. D., et al. (2008). Temporal interactions between cortical rhythms. Frontiers in Neuroscience, 2, 145-154.

Sato, W., Kochiyama, T., \& Uono, S. (2015). Spatiotemporal neural network dynamics for the processing of dynamic facial expressions. Scientific Reports, 5, 1-13.

Sato, W., Kochiyama, T., Uono, S., \& Yoshikawa, S. (2008). Time course of superior temporal sulcus activity in response to eye gaze: A combined fMRI and MEG study. Social Cognitive and Affective Neuroscience, 3, 224-232.

Sato, W., Kochiyama, T., Yoshikawa, S., Naito, E., \& Matsumura, M. (2004). Enhanced neural activity in response to dynamic facial expressions of emotion: An fMRI study. Brain Research, 20, 81-91.

Schultz, J., \& Pilz, K. S. (2009). Natural facial motion enhances cortical responses to faces. Experimental Brain Research, 194, 465-475.

Schyns, P. G., Thut, G., \& Gross, J. (2011). Cracking the code of oscillatory activity. PLoS Biology, 9, e1001064.

Singh, K. D., Barnes, G. R., Hillebrand, A., Forde, E. M. E., \& Williams, A. L. (2002). Task-related changes in cortical synchronization are spatially coincident with the hemodynamic response. Neuroimage, 16, 103-114.

Trautmann, S. A., Fehr, T., \& Herrmann, M. (2009). Emotions in motion: Dynamic compared to static facial expressions of disgust and happiness reveal more widespread emotionspecific activations. Brain Research, 1284, 100-115.

Ulloa, J. L., Puce, A., Hugueville, L., \& George, N. (2014). Sustained neural activity to gaze and emotion perception in dynamic social scenes. Social Cognitive and Affective Neuroscience, 9, 350-357.

Vuilleumier, P., \& Pourtois, G. (2007). Distributed and interactive brain mechanisms during emotion face perception: Evidence from functional neuroimaging. Neuropsychologia, 45, 174-194.

Watanabe, S., Kakigi, R., \& Puce, A. (2001). Occipitotemporal activity elicited by viewing eye movements: A magnetoencephalographic study. Neuroimage, 13, 351-363.

Watanabe, S., Miki, K., \& Kakigi, R. (2005). Mechanisms of face perception in humans: A magneto- and electroencephalographic study. Neuropathology, 25, 8-20.

Wheaton, K. J., Pipingas, A., Silberstein, R. B., \& Puce, A. (2001). Human neural responses elicited to observing the actions of others. Visual Neuroscience, 18, 401-406.

Zumer, J. M., Brookes, M. J., Stevenson, C. M., Francis, S. T., \& Morris, P. G. (2010). Relating BOLD fMRI and neural oscillations through convolution and optimal linear weighting. Neuroimage, 49, 1479-1489. 